\title{
INFANTILE STATUS EPILEPTICUS \& MESIAL TLE
}

The clinical and EEG characteristics of initial status epilepticus (SE) during infancy were studied in six patients seen in the emergency clinic between 1977 and 1988 at Tokyo Women's Medical University, Japan. Patients selected for review of medical records and laboratory findings at the time of treatment for the SE were those who subsequently developed mesial temporal epilepsy (MTLE). SE had occurred at a mean age of 14 months (range 7 months to 2 years 9 months). Two patients had developed SE during exanthem subitum. SE was characterized by fever, unilateral or generalized, prolonged seizures, SE lasting 120-380 minutes (mean $227 \mathrm{~min}$ ), postictal prolonged unconsciousness, and Todd's paresis in 3 of 6 . EEG findings were lateralized to the side of the hippocampal atrophy later confirmed by MRI, despite generalized SE. Hippocampal atrophy was thought to have preceded the SE in 2 patients. Follow-up EEGs at 6 months and after development of complex partial seizures (CPS) showed temporal spike discharges. The latency between onset of SE and CPS ranged from 3 months to 13 years 3 months. EEG temporal lobe spikes did not appear until after the onset of recurrent CPS. (Ohtsu M, Oguni H, Awaya Y, Osawa M. Clinical and EEG analysis of initial status epilepticus during infancy in patients with mesial temporal lobe epilepsy. Brain Dev June 2002;24:231-238). (Respond: Dr Mayu Ohysu, Department of Pediatrics, Tokyo Women's Medical University, 8-1 Kawada-cho, Shinjuku-ku, Tokyo 162-8666, Japan).

COMMENT. Temporal lobe epilepsy may be expected in $7.5 \%$ of infants following febrile SE, with a follow-up period of 5 to 21 years (Awaya $Y$ et al. 1992). Infants who develop hippocampal sclerosis and subsequent temporal lobe epilepsy following infantile SE have suffered a complicated and prolonged generalized or lateralized SE with fever, protracted postictal unconsciousness, lateralized EEG abnormalities, and a high incidence of Todd's paresis. The appearance of temporal lobe spikes in the EEG is delayed until after the onset of complex partial seizures.

\section{ANTIEPILEPTIC DRUG-INDUCED BONE LOSS IN YOUNG MALES}

The relationship between femoral neck bone mineral density (BMD) and antiepileptic drug therapy in ambulatory, young male patients with epilepsy (ages 25 to 54 years, mean 45 yrs) was determined in an outpatient seizure clinic at the University of Washington, Seattle. Dual-energy absorptiometry scans were obtained in 81 consecutive patients and were repeated in 54 patients, 12 to 29 months later (mean, 19 months). Nineteen patients (23\%) had suffered 25 fractures. Low BMD values were analyzed for bone loss risk factors. Age and duration of AED therapy were the 2 important risk factors associated with low femoral neck BMD. Only the youngest patients (25-44 yrs) showed significant bone loss. The mean duration of AED therapy was 18 years, and patients with continuing bone loss had received AED the shortest length of time (median 11 yrs). Phenytoin and/or carbamazepine were the most frequently used AED, in 30 and 22 of 54 patients, respectively, but no AED was specific for bone loss. Risk factors previously associated with bone loss, including vitamin D deficiency, hypocalcemia, cigarette and heavy alcohol use, were not of significance as a cause of bone loss, after correcting for age and time on AED. (Andress DL, Ozuna J, Tirschwell D et al. Antiepileptic drug-induced bone loss in young male patients who have seizures. Arch Neurol May 2002;59:781-786). (Reprints: Dennis L Andress MD, Veterans Affairs Puget Sound Health Care System (111A), 1660 S Columbian Way, Seattle, WA 98108). 
COMMENT. Young male patients taking AED therapy for seizures are at risk of significant bone loss at the femoral neck. Almost $50 \%$ have osteopenia, a 2.5 fold increased prevalence compared to the healthy male population, and the risk of fractures is increased.

\section{LEVETIRACETAM IN PARTIAL SEIZURES}

The efficacy and safety of levetiracetam (LEV) as adjunctive therapy were evaluated in 23 children (aged 6-12 years) with monotherapy-resistant partialonset seizures at Children's Hospital Medical Center, Cincinnati, OH and other major centers. Seizure frequency during an 8-week evaluation period with individualized LEV doses (20-40 mg/kg/day) was compared with the 4-week baseline seizure frequency. Seizure frequency was reduced by $>50 \%$ in $12(52 \%)$ patients. Plasma concentrations of concomitant AED (carbamazepine or valproic acid) were not affected. Adverse events included headache (33\%), anorexia (25\%), and somnolence (25\%). No alterations in mean clinical laboratory values were observed. A decrease in red blood cell count occurred in 1 patient, and thrombocytopenia in one was attributed to concomitant valproic acid treatment. (Glauser TA, Pellock JM, Bebin EM et al. Efficacy and safety of levetiracetam in children with partial seizures: an open-label trial. Epilepsia May 2002;43:518-524). (Reprints: Dr TA Glauser, Children's Hospital Medical Center, Department of Neurology OSB5, 3333 Burnet Avenue, Cincinnati, OH 45229).

COMMENT. Levetiracetam (Keppra $\left.{ }^{\circledR}\right)$, an adjunctive therapy for partial seizures in adults, is shown to be effective and relatively safe in children, by open-trial. A randomized, placebo-controlled, double-blind trial is ongoing.

In an editorial (Van Ness PC. Therapy for the epilepsies. Arch Neurol May 2002;59:732-733), Levetiracetam, introduced in 1999, is described as a broad spectrum AED, twice-daily dosing, unique mechanism of action, low toxicity, low protein binding, low risk of rash, and no drug interactions. Sedation is the main disadvantage, occurring in $15 \%$ of patients.

\section{DEGENERATIVE DISEASES}

\section{MOLECULAR DIAGNOSIS OF ALEXANDER DISEASE}

The value of clinical and MRI criteria in the diagnosis of Alexander disease was determined by using GFAP gene sequencing as the confirmatory assay in 13 patients with variable ages of onset in a study at Children's National Medical Center, Washington, DC and other centers. Genomic DNA was screened for mutations in the GFAP gene, and $12(92 \%)$ tested positive. Seven of the 12 presented in infancy (ages 2 to 18 nonths) with megalencephaly, seizures, failure to thrive, and delayed development. Five were juvenile-onset (ages 5 to 9 years) with variable symptoms, 2 being asymptomatic and the remainder with severe symptoms, including growth failure, sleepiness, and vomiting. Both groups showed progression of megalencephaly with increasing age, bulbar signs, spasticity, cognitive deficits, and developmental delay. MRI showed diffuse, symmetrical white matter abnormality in the frontal regions, sparing subcortical U-fibers. GFAP gene mutation analysis should be included in the initial diagnostic evaluation of infants or young children presenting with megalencephaly and predominantly frontal leukoencephalopathy on MRI. Gene analysis may now take the place of brain biopsy histological examination for diagnostic Rosenthal fibers. (Gorospe JR, Naidu S, Johnson AB et al. Molecular findings in symptomatic and pre-symptomatic Alexander disease patients. Neurology May (2 of 2) 